1

2

3

4

36

37

38

39

Submit to Current Opinion in Insect Science

Section: Pests and Resistance

Short Title: Rootworm Resistance to Bt Maize

\title{
Resistance to Bt Maize by Western Corn Rootworm: Insights from the Laboratory and the Field
}

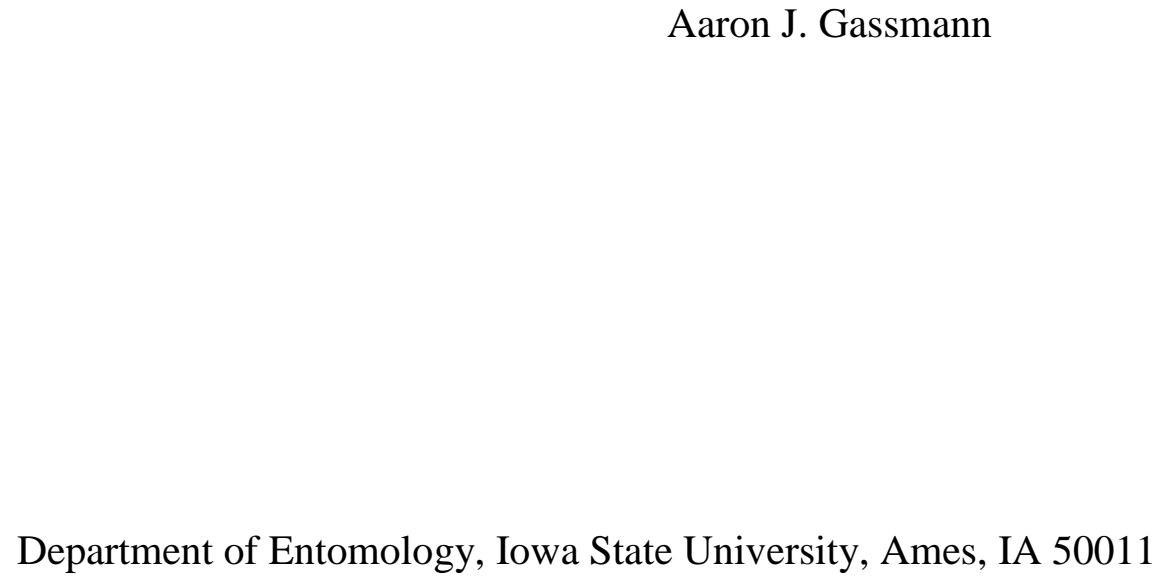




\section{Abstract}

41 Western corn rootworm is a serious pest of maize. Beginning in 2003, management of western

42 corn rootworm included transgenic maize that produces insecticidal toxins derived from the

43 bacterium Bacillus thuringiensis $(\mathrm{Bt})$. The first Bt maize hybrids produced Cry3Bb1, but

44 additional Bt toxins have since been introduced, including eCry3.1 Ab, mCry3 A and

45 Cry34/35Ab1. Laboratory selection experiments found that western corn rootworm could

46 develop resistance to all types of Bt maize following three to seven generations of selection. By

472009 cases of field-evolved resistance to Cry3Bb1 maize had been identified, with populations

48 also showing cross-resistance to mCry3A maize. Factors likely contributing to resistance were

49 the lack of a high dose of Bt toxin for maize targeting rootworm and minimal fitness costs of

50 resistance.

Introduction. The western corn rootworm, Diabrotica virgifera virgifera LeConte, is

54 among the most serious pests of maize in North America and is also a pest of maize in Europe,

55 with larval feeding on maize roots the primary cause of reduced yields [1]. As with many

56 agricultural pests, western corn rootworm has developed resistance to several pest management

57 practices, including conventional insecticides, cultural control through crop rotation, and

58 transgenic maize that produces insecticidal toxins derived from the bacterium Bacillus

59 thuringiensis $(\mathrm{Bt})$ [2-5]. Bt maize targets larval corn rootworm, and was registered for

60 management of western corn rootworm beginning in 2003, with the first Bt maize hybrids

61 producing the $\mathrm{Cry} 3 \mathrm{Bb} 1 \mathrm{Bt}$ toxin [6]. For western corn rootworm, initial attempts to manage $\mathrm{Bt}$ 
62 resistance focused on the refuge strategy, although pyramiding of multiple Bt toxins also has

63 become a common approach during the last several years.

Under the refuge strategy, non-Bt maize is grown in conjunction with Bt maize to

65 promote the survival of Bt-susceptible individuals, which may mate with Bt-resistant individuals and produce heterozygous offspring [7]. However, dispersal by adult rootworm prior to mating

67 is often limited, and this likely reduces mating between Bt-selected insects and refuge insects when refuges are spatially segregated from Bt fields [8]. Furthermore, it appears that refuge requirements often are not followed, both for the amount of refuge planted and for the proximity 70 of refuges to Bt fields [9].

When Bt crops produce a single toxin against a target pest, the risk of resistance is

72 positively associated with the survival of heterozygotes on a Bt crop (i.e., the genetic dominance

73 of resistance) $[7,10]$. As such, the refuge strategy is most effective at delaying resistance when a

74 Bt crop achieves a high dose of toxin (25 times the amount of toxin needed to kill a susceptible

75 insect) because this renders resistance a functionally recessive trait $[7,10,11]$. When resistance to

76 a Bt crop is a functionally recessive trait, both homozygous susceptible insects and heterozygous

77 insects are killed by the Bt crop. However, none of the currently registered Bt events found in

78 maize targeting western corn rootworm produce a high dose of Bt toxin $[12,13]$.

Data from Laboratory Selection Studies. For all Bt toxins used in transgenic maize targeting western corn rootworm, laboratory selection experiments have demonstrated the

81 capacity of this pest to evolve resistance rapidly when non-Bt refuges are absent. In particular, resistance to $\mathrm{Cry} 3 \mathrm{Bb} 1$ maize has been studied extensively in the laboratory.

The first such study was conducted by Meihls et al. [14], who reported similar survival 84 on $\mathrm{Cry} 3 \mathrm{Bb} 1$ maize and non-Bt maize following three generations of selection through larval 
85 development to adulthood on Cry3Bb1 maize in the greenhouse. Similar results were found for 86 additional strains of western corn rootworm [15]. Oswald et al. [16] applied incremental

87 selection, only exposing insects for a portion of the larval stage, but still found a statistically

88 significant increase in survival on Cry3Bb1 maize after four generations of selection, a

89 difference that became greater following additional generations of selection.

90 Laboratory selection of western corn rootworm for resistance to additional Bt toxins

91 provides further evidence of the capacity of this pest to develop resistance to Bt maize. This

92 includes maize producing either mCry3A, eCry3.1Ab or Cry34/35Ab1, which along with

$93 \mathrm{Cry} 3 \mathrm{Bb} 1$ maize, represents all of the currently registered Bt traits targeting western corn

94 rootworm. After seven generations of selection on mCry3A maize, using a combination of

95 maize plants in the greenhouse and seedling mats in a growth chamber, $\mathrm{LC}_{50}$ values for mCry $3 \mathrm{~A}$

96 in diet-based bioassays were significantly greater for the selected strain compared to an

97 unselected control [17]. After four generations of selection following methods similar to Meihls

98 et al. [17], no difference in survival was found between eCry3.1Ab maize and non-Bt maize for

99 the selected strain, but survival was significantly lower on eCry3.1Ab maize compared to non-Bt

100 maize for the control strain [18]. Laboratory selection for resistance to Cry34/35Ab1 maize in

101 two strains caused a statistically significant increase in the level of resistance by both strains

102 following one generation of selection on Cry34/35Ab1 maize [19]. However, the response to

103 additional bouts of selection appeared to be modest, and after 10 generations of selection,

104 survival of the selected strains on Cry34/35Ab1 maize was between 10 and 20\% compared to ca.

$10580 \%$ for the unselected strain on non-Bt maize [19]. A separate experiment, using a different

106 strain of western corn rootworm, found a response to selection after four generations of selection

107 on Cry34/35Ab1 maize [20]. 
Field-Evolved Resistance. Field-evolved resistance to Bt maize by western corn

rootworm was observed in Iowa, USA, beginning in 2009 [21]. Resistance was found first to $\mathrm{Cry} 3 \mathrm{Bb} 1$ maize, and these cases of resistance occurred 7 years after the registration of Cry3Bb1 maize [6]. Initially, field-evolved resistance was found to be incomplete, with survival on Cry3Bb1 maize less than survival on non-Bt maize. Maize fields with Cry3Bb1-resistant western corn rootworm were associated with continuous cultivation of maize (i.e., at least 7 consecutive years) and the cultivation of Cry3Bb1 maize for 3 to 6 consecutive years. These initial cases of $\mathrm{Cry} 3 \mathrm{Bb} 1$ resistance were detected because of observations made in the field by farmers and crop consultants of high levels of feeding injury to Cry3Bb1 maize. Root injury among these fields was found to be greater than 1.5 nodes, with the loss of one node of roots translating to a 15 to $17 \%$ reduction in yield $[22,23]$. The detection of resistance based on reports of pest injury to a Bt crop suggests that for pests with localized dispersal, such as western corn rootworm, crop injury and high pest abundance in the field may provide a more informative initial warning about the onset of resistance than can be achieved through routine monitoring of haphazardly selected pest populations [12].

Additional fields with Cry3Bb1-resistant western corn rootworm were found in Iowa during 2010 and 2011 [5,24]. Unlike populations sampled in 2009, mean survival among field populations did not differ between $\mathrm{Cry} 3 \mathrm{Bb} 1$ maize and non-Bt, indicating complete resistance to Cry3Bb1 maize. Additionally, the level of resistance to Cry3Bb1 maize found among field populations increased significantly between 2009 and 2011 [5]. Results of a field experiment showed no difference in survival of western corn rootworm on Cry3Bb1 maize and non-Bt maize in two fields where resistance had been identified with bioassays [13]. Similar to what was found in Iowa, field-evolved resistance to Cry3Bb1 maize was found for populations of western 
131 corn rootworm in Nebraska that were sampled in 2011 and 2012 from fields characterized by

132 continuous cultivation of Cry3Bb1 maize and greater than one node of feeding injury to

133 Cry3Bb1 maize [25].

134 Initial cases of field-evolved resistance displayed a common pattern of association with

135 continuous maize cultivation and continuous planting of Cry3Bb1 maize [21,25]. However, over

136 time, pest dispersal also appeared to become important because some fields with Cry3Bb1

137 resistance sampled in Iowa during 2012 had only been planted to maize for 2 years [5].

138 Additionally, while Cry3Bb1 maize was the primary type of maize grown in fields where

139 resistance was detected, resistance also was found to mCry3A maize, implying cross-resistance

140 between mCry3A and Cry3Bb1 [5,25]. The high degree of structural similarity between these Bt

141 toxins suggests a similar mode of action for mCry3A and Cry3Bb1, which are both three-domain

142 Bt toxins, and this likely facilitated the development of cross-resistance [5,26,27].

143 Inheritance of Resistance and Fitness Costs. Because Bt maize targeting western corn

144 rootworm does not achieve a high-dose (i.e., does not produce 25 times more Bt toxin than is

145 required to kill susceptible individuals [11]), the inheritance of resistance traits for survival on $\mathrm{Bt}$

146 maize may be non-recessive [7]. Computer modeling suggests that the rate at which pests adapt

147 to Bt crops is greater when resistance is non-recessive [10]. Experimental work to date has

148 found evidence of non-recessive inheritance of resistance to Cry3Bb1 maize. In a study with

149 laboratory-selected strains, larval survival on Cry3Bb1 maize was significantly greater for a

150 heterozygous strain and a Cry3Bb1-resistant strain compared to the susceptible strain [14].

151 Another study, using a different laboratory-selected strain with Cry3Bb1 resistance, found

152 similar larval survival on Cry3Bb1 maize for the resistant strain and one of the heterozygous

153 strains (susceptible female $\times$ resistant male). However, survival for the other heterozygous strain 
154 (resistant female $\times$ susceptible male) was not statistically different from the susceptible strain, 155 suggesting sex linkage for resistance in this strain [28]. In a study of two laboratory strains with 156 field-derived resistance to Cry3Bb1 maize, survival to adulthood on Cry3Bb1 maize seedling 157 mats for heterozygous strains was intermediate between resistant and susceptible strains in one 158 case but did not differ statistically from the susceptible strain in the other case. In general, 159 resistance to $\mathrm{Cry3Bb} 1$ maize by western corn rootworm tends to display inheritance values that 160 are between recessive and additive, which is consistent with theoretical predictions based on 161 survival of susceptible strains on $\mathrm{Cry} 3 \mathrm{Bb} 1$ maize. This non-recessive inheritance of resistance 162 likely contributed to the rapid development of Cry3Bb1 resistance by western corn rootworm in 163 the laboratory and field.

164 Fitness costs of Bt resistance are important because they can delay the evolution of 165 resistance when non-Bt refuges are present by removing resistance alleles from refuge 166 populations [29]. Additionally, fitness costs can lead to a reduction in resistance allele frequency 167 once selection is removed. Several studies have tested for fitness costs of resistance to Cry3Bb1 168 maize by western corn rootworm, and most of these have used strains with laboratory-selected 169 resistance. A comparison of three non-diapausing Cry3Bb1-resistant strains with companion 170 non-diapausing susceptible strains revealed no fitness costs in both a greenhouse study and a 171 field study that measured larval survival and size [15]. However, in a third experiment that 172 included both a greenhouse and laboratory component and measured several life-history traits 173 (i.e., developmental rate, survival to adulthood, adult longevity, egg viability and fecundity), an 174 overall reduction in fecundity was found among Cry3Bb1-resistant strains [15]. Although each 175 pair of resistant and susceptible strains were started from the same parental strain, the resistant 176 strains were never backcrossed to their respective susceptible strains during selection for 
177 resistance, which may have introduced other genetic differences that confounded the assessment

178 of fitness costs in this experiment [15]. A separate study using a different set of laboratory-

179 selected strains did not detect any fitness costs of Cry3Bb1 resistance across several life-history

180 traits, including developmental rate, survival to adulthood, adult longevity, fecundity and egg

181 viability [30]. Additional experiments using one of the strains studied by Oswald et al. [30]

182 found that the presence of entomopathogenic fungi and nematodes failed to impose a fitness cost 183 of higher mortality for Cry3Bb1-resistant western corn rootworm [28,31]. Also, across three 184 different non-Bt maize lines, in an experiment that included both a greenhouse and laboratory 185 component, fitness costs were not detected for several life history characteristics, including larval 186 developmental rate, survival to adulthood, adult size and longevity, and fecundity [32].

In a study of two strains with field-derived resistance, data were collected on fitness costs affecting developmental rate, survival to adulthood, adult size and lifespan, fecundity and egg 189 viability [33]. For one strain fitness costs were absent, however, for the other strain, fitness costs 190 were found for developmental rate, survival to adulthood and fecundity [33]. In general, it appears that fitness costs of resistance are often absent, but can arise in some cases, and in 192 particular appear to affect fecundity. However, over time, natural selection may favor those resistance traits that have the fewest accompanying fitness costs, and such resistance traits would 194 be the most likely to persist in populations once selection is removed. toxin, the magnitude of resistance for traits conferring survival on Bt crops is often far greater than observed for Bt maize targeting western corn rootworm. For example, a three to six fold increase in resistance by western corn rootworm to Bt maize is associated with severe feeding injury to Bt maize in the field, and similar survival on Bt and non-Bt maize $[5,13,21]$. By contrast, resistance ratios to Cry1Ac cotton for a pest such as pink bollworm, Pectinophora 
201

202

203

204

205

206

207

208

209

210

211

212

213

214

215

216

217

218

219

220

221

222

223

224

gossypiella (Saunders), for which Bt cotton represents a high-dose Bt crop, may be one to two orders of magnitude greater [5]. Additionally, resistance to Cry1Ac cotton by pink bollworm is associated with a recessively inherited, monogenic trait conferred by truncation of a cadherin protein, which is a mid-gut protein involved in the mode of action for some Bt toxins $[34,35]$. Although little is currently known about the mechanism of Bt resistance in western corn rootworm, it may differ from resistance to high-dose Bt crops in that it is not conferred by an allele of large effect, and at least some of the loci for resistance may contain alleles that show non-recessive inheritance. Work by Flagel et al. [36] identified a genetic marker associated with resistance to $\mathrm{Cry} 3 \mathrm{Bb} 1$ in western corn rootworm. While primarily homozygous resistant individuals survived exposure to Cry3Bb1, some survival of heterozygotes also was observed. It should be noted that this genetic marker explained less than $25 \%$ of the genetic variation in resistance, implying the presence of additional loci conferring resistance. The survival of heterozygous resistant larvae in plant-based bioassays provides additional evidence for nonrecessive inheritance of some resistance alleles [14,33]. Additionally, the incremental response to selection for resistance to Bt maize by western corn rootworm $[14,16,19,20]$ is consistent with a phenotypic trait that has a polygenic genetic architecture [37].

Additional Considerations. Structural similarities between Bt toxins may have contributed to cross-resistance between Cry3Bb1 maize and mCry3A maize [5,27]. Thus, crossresistance may extend to additional three domain Bt toxins that possess structural homologies to Cry3Bb1 and mCry3A. Additionally, pyramiding of Cry34/35Ab1 with either mCry3A or Cry3Bb1 to manage Cry3-resistant populations of western corn rootworm will place intense selection on pest populations to develop resistance to the structurally dissimilar Cry34/35Ab1 toxin, for which cross resistance has not been detected [5]. The use of RNA interference (RNAi) likely represents the next transgenic approach for management of western corn rootworm [38]. To the 
225 extent that RNAi will be pyramided with Bt traits for management of corn rootworm, it remains

226 unclear to what degree past selection for resistance to Bt toxins may complicate future

227 management of western corn rootworm.

228

229

Acknowledgements

I thank Aubrey Paolino for assistance with constructing the figure in this paper, and Eric

231 Clifton, Kenneth Masloski, Aubrey Paolino, Ram Shrestha and Coy St. Clair for comments on

232 this work. This work was supported by the Agricultural Experiment Station at Iowa State

233 University and by Biotechnology Risk Assessment Grant Program competitive grant no. 2012-

$234 \quad 33522-20010$ from the USDA National Institute of Food and Agriculture.

235

236

References

237

238

239

240

241

242

243

1. Gray ME, Sappington TW, Miller NJ, Moeser J, Bohn MO: Adaptation and invasiveness of western corn rootworm: intensifying research on a worsening pest. Annu Rev Entomol 2009, 54:303-321.

2. Spencer JL, Hughson SA, Levine E: Insect resistance to crop rotation. In Insect Resistance Management: Biology, Economics and Predictions. Edited by Onstad DW: Academic Press; 2014, 233-278.

244 3. Meinke LJ, Siegfried BD, Wright RJ, Chandler LD: Adult susceptibility of Nebraska 245 western corn rootworm (Coleoptera: Chrysomelidae) populations to selected insecticides. J Econ Entomol 1998, 91:594-600.

247 4. Pereira AE, Wang H, Zukoff SN, Meinke LJ, French BW, Siegfried BD: Evidence of field248 evolved resistance to bifenthrin in western corn rootworm (Diabrotica virgifera 
virgifera LeConte) populations in western Nebraska and Kansas. PLoS ONE 2015, 10(11):e0142299. DOI:10.1371/journal.pone.0142299.

- 5. Gassmann AJ, Petzold-Maxwell JL, Clifton EH, Dunbar MW, Hoffmann AM, Ingber DA, Keweshan RS: Field-evolved resistance by western corn rootworm to multiple Bacillus thuringiensis toxins in transgenic maize. Proc Natl Acad Sci U S A 2014, 111:5141-5146.

Demonstrates cross-resistance between Cry3Bb1 maize and mCry3A maize among field populations of western corn rootworm

6. EPA [Environmental Protection Agency]: Biopesticides Registration Action Document: Bacillus thuringiensis Cry3Bb1 Protein and the Genetic Material Necessary for Its Production (Vector PV-ZMIR13L) in MON 863 Corn (OECD Unique Identifier: MON-ØØ863-5). http://www3.epa.gov/pesticides/chem_search/reg_actions/pip/cry3bb1-brad.pdf (accessed 4 January, 2016); 2010.

7. Gould F: Sustainability of transgenic insecticidal cultivars: integrating pest genetics and ecology. Аппи Rev Entomol 1998, 43:701-726.

8. Hughson SA, Spencer JL: Emergence and abundance of western corn rootworm in Bt cornfields with structured and seed blend refuges. J Econ Entomol 2015, 108:114125.

9. Jaffe G: Complacency on the Farm: Significant Noncompliance with EPA's Refuge Requirements Threatens the Future Effectiveness of Genetically Engineered Pestprotected Corn. Washington, DC: Center for Science in the Public Interest; 2009. 
271 10. Tabashnik BE, Gassmann AJ, Crowder DW, Carrière Y: Insect resistance to Bt crops:

272 evidence versus theory. Nat Biotechnol 2008, 26:199-202.

273 11. EPA [Environmental Protection Agency]: Final report of the FIFRA scientific advisory

274 panel subpanel on Bacillus thuringiensis $(B t)$ plant-pesticides and resistance

275

- 12. Andow DA, Pueppke SG, Schaafsma AW, Gassmann AJ, Sappington TW, Meinke LJ, management. http://archive.epa.gov/scipoly/sap/meetings/web/pdf/finalfeb.pdf (Accessed 7 January, 2016); 1998.

Provides a new framework for detecting and managing resistance to Bt maize by western corn 282 rootworm.

13. Gassmann AJ: Field-evolved resistance to Bt maize by western corn rootworm: predictions from the laboratory and effects in the field. J Invertebr Pathol 2012, 110:287-293.

14. Meihls LN, Higdon ML, Siegfried BD, Miller NJ, Sappington TW, Ellersieck MR, Spencer TA, Hibbard BE: Increased survival of western corn rootworm on transgenic corn within three generations of on-plant greenhouse selection. Proc Natl Acad Sci U S A 2008, 105:19177-19182.

15. Meihls LN, Higdon ML, Ellersieck MR, Tabashnik BE, Hibbard BE: Greenhouse-selected resistance to Cry3Bb1-producing corn in three western corn rootworm populations. PLoS ONE 2012, 7(12):e51055. doi:10.1371/journal.pone.0051055. 
16. Oswald KJ, French BW, Nielson C, Bagley M: Selection for Cry3Bb1 resistance in a genetically diverse population of nondiapausing western corn rootworm (Coleoptera: Chrysomelidae). J Econ Entomol 2011, 104:1038-1044.

17. Meihls LN, Higdon ML, Ellersieck M, Hibbard BE: Selection for resistance to mCry3Aexpressing transgenic corn in western corn rootworm. J Econ Entomol 2011, 104:1045-1054.

18. Frank DL, Zukoff A, Barry J, Higdon ML, Hibbard BE: Development of resistance to eCry3.1Ab-expressing transgenic maize in a laboratory-selected population of western corn rootworm (Coleoptera: Chrysomelidae). J Econ Entomol 2013, 106:2506-2513.

19. Lefko SA, Nowatzki TM, Thompson SD, Binning RR, Pascual MA, Peters ML, Simbro EJ, Stanley BH: Characterizing laboratory colonies of western corn rootworm (Coleoptera: Chrysomelidae) selected for survival on maize containing event DAS59122-7. J Appl Entomol 2008, 132:189-204.

- 20. Deitloff J, Dunbar MW, Ingber DA, Hibbard BE, Gassmann AJ: Effects of refuges on the evolution of resistance to transgenic corn by the western corn rootworm, Diabrotica virgifera virgifera LeConte. Pest Manag Sci 2016, 72:190-198.

Shows resistance to Cry34/35Ab1 maize under laboratory selection and demonstrates the potential for non-Bt refuges to delay resistance.

21. Gassmann AJ, Petzold-Maxwell JL, Keweshan RS, Dunbar MW: Field-evolved resistance to Bt maize by western corn rootworm. PLOS ONE 2011, 6(7):e22629. doi:22610.21371/journal.pone.0022629. 
22. Tinsley NA, Estes RE, Gray ME: Validation of a nested error component model to estimate damage caused by corn rootworm larvae. J Appl Entomol 2013, 137:161169.

23. Dun Z, Mitchell PD, Agosti M: Estimating Diabrotica virgifera virgifera damage functions with field trial data: applying an unbalanced nested error component model. J Appl Entomol 2010, 134:409-419.

24. Gassmann AJ, Petzold-Maxwell JL, Keweshan RS, Dunbar MW: Western corn rootworm and Bt maize: challenges of pest resistance in the field. GM Crops and Food 2012, 3:235-244.

-25. Wangila DS, Gassmann AJ, Petzold-Maxwell JL, French BW, Meinke LJ: Susceptibility of Nebraska western corn rootworm populations (Coleoptera: Chrysomelidae) populations to Bt corn events. J Econ Entomol 2015, 108:742-751.

Provides additional documentation of resistance to Cry3Bb1 maize and mCry3A maize and cross-resistance between these Bt toxins.

26. Tabashnik BE, Gould F: Delaying corn rootworm resistance to Bt corn. J Econ Entomol 2012, 105:767-776.

27. Carriere Y, Crickmore N, Tabashnik BE: Optimizing pyramided transgenic Bt crops for sustainable pest management. Nat Biotechnol 2015, 33:161-168.

28. Petzold-Maxwell JL, Cibils-Stewart X, French BW, Gassmann AJ: Adaptation by western corn rootworm (Coleoptera: Chrysomelidae) to Bt maize: inheritance, fitness costs, and feeding preference. $J$ Econ Entomol 2012, 105:1407-1418.

29. Gassmann AJ, Carrière Y, Tabashnik BE: Fitness costs of insect resistance to Bacillus thuringiensis. Aппи Rev Entomol 2009, 54:147-163. 
30. Oswald KJ, French BW, Nielson C, Bagley M: Assessment of fitness costs in Cry3Bb1resistant and susceptible western corn rootworm (Coleoptera: Chrysomelidae) laboratory colonies. J Appl Entomol 2012, 136:730-740.

31. Hoffmann AM, French BW, Jaronski ST, Gassmann AJ: Effects of entomopathogens on mortality of western corn rootworm and fitness costs of resistance to Cry3Bb1 maize. J Econ Entomol 2014, 107:352-360.

32. Hoffmann AM, French BW, Hellmich RL, Lauter N, Gassmann AJ: Fitness costs of resistance to Cry3Bb1 maize by western corn rootworm. J Appl Entomol 2015, 139:403-415.

33. Ingber DA, Gassmann AJ: Inheritance and fitness costs of resistance to Cry3Bb1 corn by western corn rootworm (Coleoptera: Chrysomelidae). J Econ Entomol 2015, 108:2421-2432.

34. Tabashnik BE, Biggs RW, Higginson DM, Henderson S, Unnithan DC, Unnithan GC, EllersKirk C, Sisterson MS, Dennehy TJ, Carrière Y, et al.: Association between resistance to Bt cotton and cadherin genotype in pink bollworm. J Econ Entomol 2005, 98:635644.

35. Morin S, Biggs RW, Sisterson MS, Shriver L, Ellers-Kirk C, Higginson D, Holley D, Gahan LJ, Heckel DG, Carrière Y, et al.: Three cadherin alleles associated with resistance to Bacillus thuringiensis in pink bollworm. Proc Natl Acad Sci U S A 2003, 100:50045009.

- 36. Flagel LE, Swarup S, Chen M, Bauer C, Wanjugi H, Carroll M, Hill P, Tuscan M, Bansal R, Flannagan R, et al.: Genetic markers for western corn rootworm resistance to Bt toxin. G3 2015, 5:399-405. 
361 Identifies a genomic region and associated genetic marker linked with Cry3Bb1 resistance in

362 western corn rootworm

363 37. Futuyma DJ: Evolutionary Biology. Sunderland, MA: Sinauer Associates, Inc.; 1998.

364 38. Baum JA, Bogaert T, Clinton W, Heck GR, Feldmann P, Ilagan O, Johnson S, Plaetinck G, 365 Munyikwa T, Pleau M, et al.: Control of coleopteran insect pests through RNA 366 interference. Nat Biotechnol 2007, 25:1322-1326.

367 


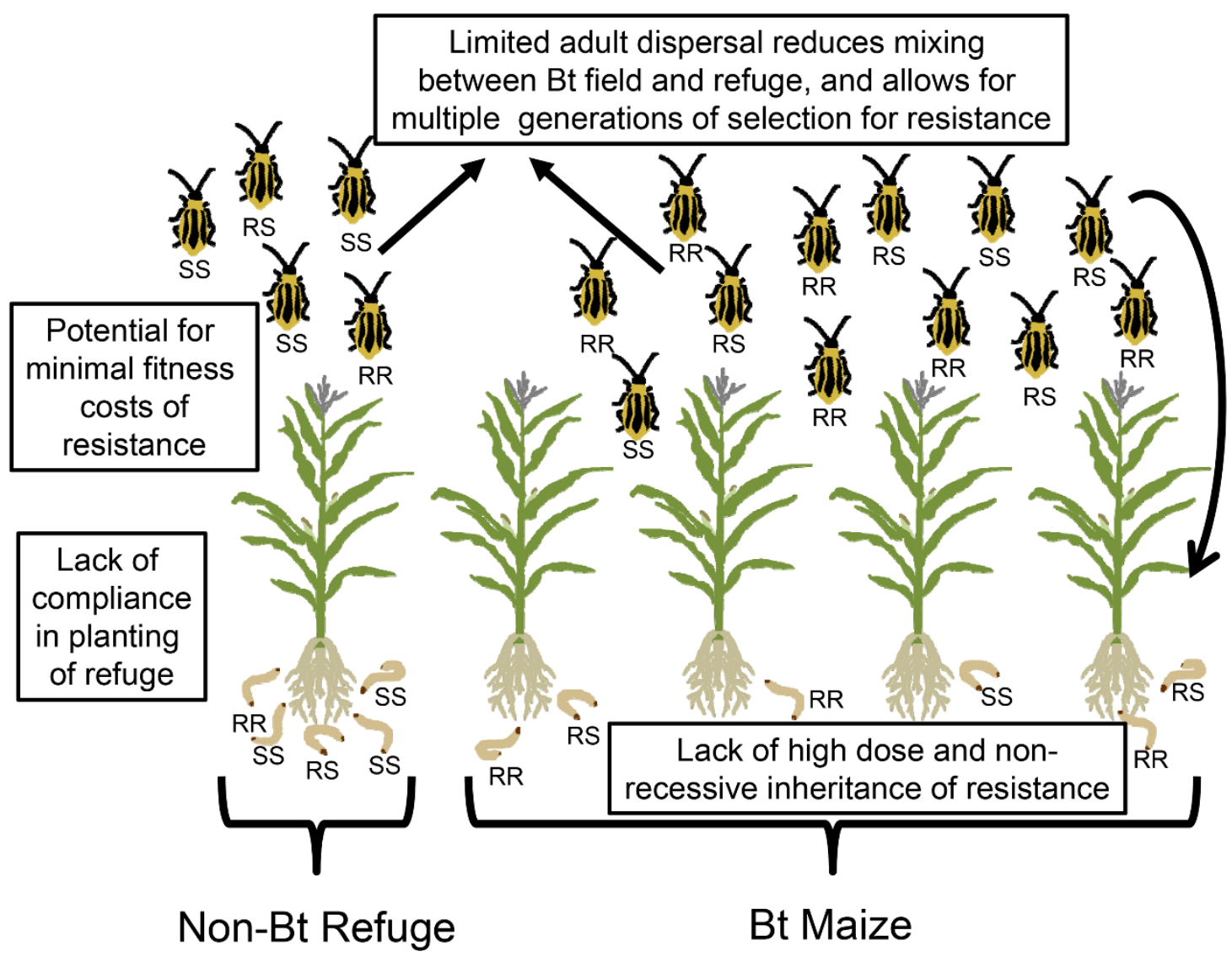

Fig. 1 Factors contributing to Bt resistance by western corn rootworm. The genotype is displayed below each larval and adult insect as RR = homozygous resistant, $\mathrm{RS}=$ heterozygous, and SS = homozygous susceptible. A lack of fitness costs contributes to the survival of resistant and heterozygous individuals in the refuge, and a lack of a high dose of Bt toxin permits the survival of homozygous susceptible and heterozygous individuals on Bt maize. 